\title{
$\mathrm{J}$

\section{Lyotropic Smectic B Phase Formed in Suspensions of Charged Colloidal Platelets}

\author{
Dzina Kleshchanok, ${ }^{*}{ }^{\dagger}$ Peter Holmqvist, ${ }^{\ddagger}$ Janne-Mieke Meijer, ${ }^{\dagger}$ and Henk N. W. Lekkerkerker ${ }^{\dagger}$ \\ ${ }^{\dagger}$ Van’t Hoff Laboratory, Debye Institute for Nanomaterials Science, Utrecht University, The Netherlands \\ ${ }^{\ddagger}$ Soft Condensed Matter Group, Institute of Complex Systems (ICS-3), Forschungszentrum Juelich, Germany
}

ABSTRACT: Here, we present the first observation of a smectic B $\left(\mathrm{Sm}_{\mathrm{B}}\right)$ phase in a system of charged colloidal gibbsite platelets suspended in dimethyl sulfoxide (DMSO). The use of DMSO, a polar aprotic solvent, leads to a long range of the electrostatic Coulomb repulsion between platelets. We believe this to be responsible for the formation of the layered liquid crystalline phase consisting of hexagonally ordered particles, that is, the $\mathrm{Sm}_{\mathrm{B}}$ phase. We support our finding by highresolution $\mathrm{X}$-ray scattering experiments, which additionally indicate a high degree of ordering in the $\mathrm{Sm}_{\mathrm{B}}$ phase.

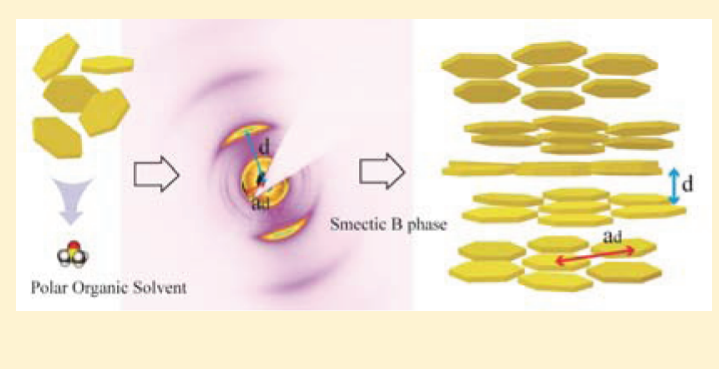

\section{INTRODUCTION}

Self-organization in colloidal suspensions leads to a fascinating range of colloidal crystal and liquid crystalline (LC) phases. ${ }^{1,2}$ Initially attention of both experiments and simulations was focused on spherical particles interacting through hard-core repulsion. $^{3-5}$ Subsequently, these studies were extended to colloids with anisotropic shapes, such as rods and plates, ${ }^{6,7}$ and also to a variety of colloidal interactions. ${ }^{8}$ Attraction (depletion, ${ }^{9-12}$ van der Waals, ${ }^{13,14}$ or Coulomb ${ }^{13,15}$ ) and/or repulsion ( steric $^{16}$ or Coulomb ${ }^{17}$ ) were applied to govern the colloidal self-assembly process. Additionally, recognition mechanisms based on particles with complementary shapes ${ }^{18}$ or on Watson-Crick attraction between DNA strands ${ }^{19-22}$ have led to an extended control over the self-organization process.

LC phase formation in suspensions of hard colloidal discs has been studied theoretically, ${ }^{23,24}$ experimentally ${ }^{25}$ and using computer simulations. ${ }^{26}$ Already in the 1940s Lars Onsager qualitatively predicted a transition from a disordered isotropic (I) to an orientationally ordered nematic (N) phase in suspensions of hard platelet-like particles. ${ }^{27}$ At higher colloidal concentrations, platelets can also form a columnar (C) phase, with orientational and 2D positional ordering. Experimentally, both LC phases ( $\mathrm{N}$ and $\mathrm{C}$ ) were found only in a single hard platelet system, namely, that of sterically stabilized gibbsite $(\gamma$ $\left.\mathrm{Al}(\mathrm{OH})_{3}\right)$ platelets. $^{25}$

Charged colloidal platelets have been studied mostly experimentally. In addition to the typical for platelets I-N-C phase sequence, a columnar nematic ${ }^{28}$ and lamellar ${ }^{29-32}$ phases were observed. Moreover, even chiral liquid crystals were found in aqueous suspensions of graphehe oxide sheets. ${ }^{33}$ The enrichment of the phase diagram of charged platelets is clearly due to the electrostatic repulsion between the platelets. Recently, Morales-Anda et al. ${ }^{34}$ presented Monte Carlo computer simulation results for a model system of charged colloidal platelets. While the authors predict a columnar nematic phase characterized by interpenetrating columns, no evidence for any layered LC phase, such as smectic or lamellar, in charged platelet suspensions was found.

In this article, we show that increasing the range of the electrostatic Coulomb repulsion leads to the formation of a new and unexpected LC phase in colloidal platelet suspensions. Specifically, we study monodisperse rigid gibbsite platelets with a diameter of $200 \mathrm{~nm}$ and a thickness of $10 \mathrm{~nm}$ and demonstrate that, under strong long ranged repulsion, a smectic $\mathrm{B}\left(\mathrm{Sm}_{\mathrm{B}}\right)$ phase is formed. The $\mathrm{Sm}_{\mathrm{B}}$ phase belongs to the family of ordered smectics, where the particle directors are oriented along the layer's normal and particles themselves exhibit hexagonal ordering within the layers. ${ }^{35}$ This rare phase so far has been exclusively observed in the thermotropic liquid crystal world of rod-like mesogens. ${ }^{35}$ Recent research also indicates its presence in colloidal suspensions of charged sedimenting silica rods. ${ }^{36}$

Our strategy for obtaining a hexagonally ordered smectic phase lied in using platelets with a high diameter/thickness (D/ $L$ ) ratio, that interact via long-range repulsion. For this purpose, we needed a polar solvent that provides charge stabilization of the gibbsite. However, it should differ from water, as solvent self-ionization and contamination by residual $\mathrm{CO}_{2}$ should not occur. With such a solvent, very low-ionic strength systems can easily be achieved. Thus, we chose to disperse the gibbsite platelets in DMSO, a polar aprotic, and low-viscous solvent of dielectric constant $\varepsilon=47.2$, at $T=293 \mathrm{~K}$, and viscosity $\eta_{0}=2 \times$ $10^{-3} \mathrm{~Pa} \cdot \mathrm{s}$. Here, we demonstrate the first experimental findings of the $\mathrm{Sm}_{\mathrm{B}} \mathrm{LC}$ phase in suspensions of colloidal platelets. Our finding is supported by high-resolution X-ray scattering experiments on suspensions over a wide platelet concentration range. Furthermore, the scattering data show high ordering of the smectic phase and verify that the observed phase transition

Received: January 17, 2012

Published: March 6, 2012 
happened in equilibrium and was not affected by sedimentation.

\section{RESULTS AND DISCUSSION}

Synthetic and Experimental Details. Colloidal gibbsite platelets used in this study were synthesized by hydrothermal treatment of aluminum alkoxides (aluminum-iso-propoxide and aluminum-sec-butoxide) in acidic environment as described in detail elsewhere. ${ }^{37}$ To provide additional stabilization, $\mathrm{Al}_{13}$ polycations (as produced by hydrolysis of aluminum chlorohydrate, $\mathrm{Al}_{2}(\mathrm{OH})_{5} \mathrm{Cl}_{2} \cdot 3 \mathrm{H}_{2} \mathrm{O}$, Locron $\mathrm{P}$, Clariant) were adsorbed onto the gibbsite surfaces. Through a sequence of centrifugations $(15 \mathrm{~h}, 1200 \mathrm{G})$ and redispersions, excess of $\mathrm{Al}_{13}$ polycations was removed and the platelets were transferred to DMSO. Gibbsite is stable in DMSO due to the electrostatic repulsion between the platelets. The average particle diameter, $\langle D\rangle$, the thickness, $\langle L\rangle$, the standard deviation, $\sigma$, and polydisersity index, PDI, were determined from TEM and AFM images and are listed in Table 1. Figure 1 shows a TEM image of the gibbsite platelets.

Table 1. Characteristic Sizes of Gibbsite Platelets As Determined from TEM and AFM

\begin{tabular}{ccccccc} 
& $<D>/ \mathrm{nm}$ & $\sigma_{D} / \mathrm{nm}$ & $\mathrm{PDI}_{D} / \%$ & $<L>/ \mathrm{nm}$ & $\sigma_{L} / \mathrm{nm}$ & $\mathrm{PDI}_{L} / \%$ \\
Gibbsite & 218.4 & 34.5 & 15.8 & 7.9 & 2.0 & 24.8 \\
\hline
\end{tabular}

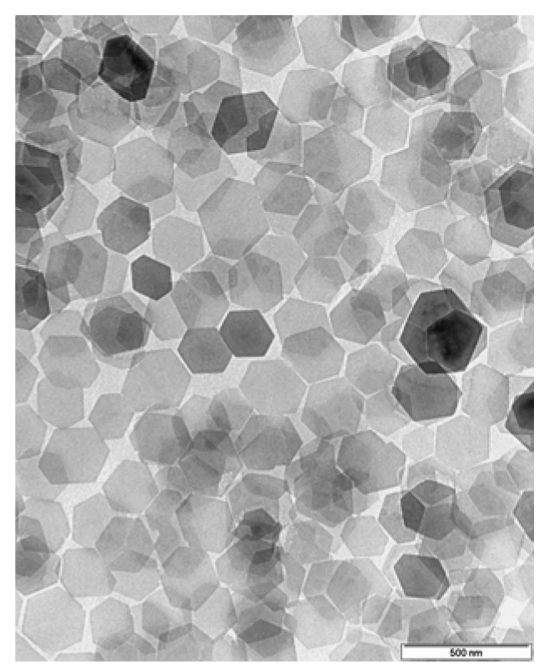

Figure 1. TEM image of gibbsite $\left(\gamma-\mathrm{Al}(\mathrm{OH})_{3}\right)$ platelets. Scale bar indicates $500 \mathrm{~nm}$.

To verify that our system was in equilibrium, that is, not kinetically arrested or affected by sedimentation, we used the following approach. After prolonged mechanical stirring, all samples were stored vertically at $20{ }^{\circ} \mathrm{C}$ to reach phase equilibrium. The macroscopic phase separation took $24 \mathrm{~h}$, after which the phase ratio did not change anymore. We waited at least $96 \mathrm{~h}$ before any measurements were performed. Later, sedimentation was not observed for another month. The X-ray scattering experiments indicated that no kinetic arrest had occurred.

Birefringence of the samples was checked with crossed polarizers, and when found present, this indicated liquid crystallinity. To determine the structure of the LC samples, we performed X-ray scattering experiments at the European Synchrotron Radiation Facility (ESRF) in Grenoble, France, at the Dutch-Belgian beamline BM-26. ${ }^{38}$ A microradian X-ray diffraction setup was used. ${ }^{39}$ This setup provided a range of scattering vector, $q$, values of $0.011 \leq q \leq 0.370 \mathrm{~nm}^{-1}$. The modulus of the scattering vector is determined by the scattering angle $2 \theta$ as $q=4 \pi \sin \theta / \lambda$.

Identification of the LC Smectic B Phase. Figure 2a presents a $2 \mathrm{D}$-scattering pattern of a sample with $4.8 \mathrm{vol} \%$ gibbsite platelets in DMSO. The sample is in a LC state. The scattering pattern is very anisotropic, showing several peaks. The peaks at small and large $q$ vectors (denominated $q_{\mathrm{S}}$ and $q_{\mathrm{L}}$, respectively) dominate the scattering normal to each other, indicating that the structures at the two length scales are oriented perpendicular to each other. Similar scattering patterns were obtained at different positions in the capillaries and for all investigated gibbsite volume fractions above the disordered phase. The $2 \mathrm{D}$ scattering pattern was observed to rotate depending on the position in the capillary. However, peak positions always remained the same. Therefore, the differences in scattering patterns are not due to structural rearrangement of the LC phase but arise from different orientations of the LC domains. Since only one or, in rare cases, two overlaying scattering patterns from the ordered phase were present during one measurement, the size of the structural domains should be larger than the beam size, which was approximately $0.5 \mathrm{~mm}$ in diameter.

To investigate the structure at the two length scales, we extracted the intensity profile for the $q_{\mathrm{S}}$ and $q_{\mathrm{L}}$ wedge. Figure $2 \mathrm{~b}$ shows the extracted $q_{\mathrm{L}}$ intensity profile for the suspension with 8.9 vol\% of gibbsite. There are two remarkable features in this scattering pattern. First, the strong sharpness of the first scattering peak, identified here as the (001) peak. Second, the number of clear and well-defined peaks of higher order, $(00 n)$ (where $n \geq 2$ ), that are found. The peaks positions have a 1:2:3:4 relation, indicative of lamellar and smectic ordering. This means that the ordered phase has large two-dimensional layers periodically stacked after each other. The layers are orientated over the whole crystal domain, as can be concluded from the very anisotropic 2D-scattering pattern and the appearance of several sharp higher order $(00 n)$ peaks. The interlayer distance, $d$, calculated from the $q_{001}$ value as $d=2 \pi$ / $q_{(001)}$, is $100 \mathrm{~nm}$, which is significantly larger than the thickness of the platelet $(L=7.9 \mathrm{~nm})$ due to the long-ranged electrostatic repulsion between platelets faces.

In the $q_{\mathrm{S}}$ direction, several peaks with the relative position of $1: \sqrt{ } 3: \sqrt{ } 4: \sqrt{ } 7$ are found, as seen in Figure $2 c$. These peaks can be attributed to the (100), (110), (200), and (210) Bragg reflections. This indicates that the sample has a hexagonal structure in this direction. The hexagonal lattice spacing, $a_{\mathrm{D}}$, was calculated by plotting $q_{h k l}$ values ( $h k l$ the Miller indices) of the Bragg peaks versus $\left(h^{2}+h k+k^{2}\right)^{1 / 2}$ (see the inset of Figure $2 c)$. For a hexagonal structure, such a plot passes through the origin and has a linear slope, ${ }^{40}$ related to a lattice spacing, $a_{\mathrm{D}}$. The value of $a_{\mathrm{D}}$ was found to be $284.1 \mathrm{~nm}$. It means the distance between platelets edges is about $65 \mathrm{~nm}$. It is clear that the distance between platelets within layers is significantly smaller than the interlayer distance. This indicates that the strength of the electrostatic repulsion is smaller between the platelets in the layer than between the layers.

We conclude that the structure of the LC ordered gibbsite/ DMSO samples is composed of planes constructed of hexagonally ordered platelets. In other words, this is a $\mathrm{Sm}_{\mathrm{B}}$ phase. To our knowledge, this is the first time this structure has been observed in a colloidal platelet dispersion. 


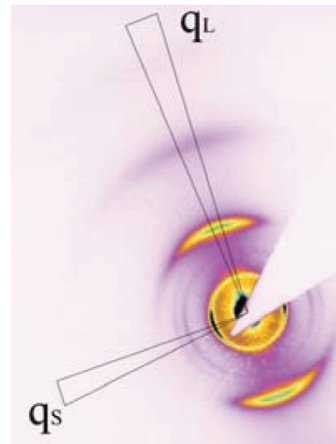

a)
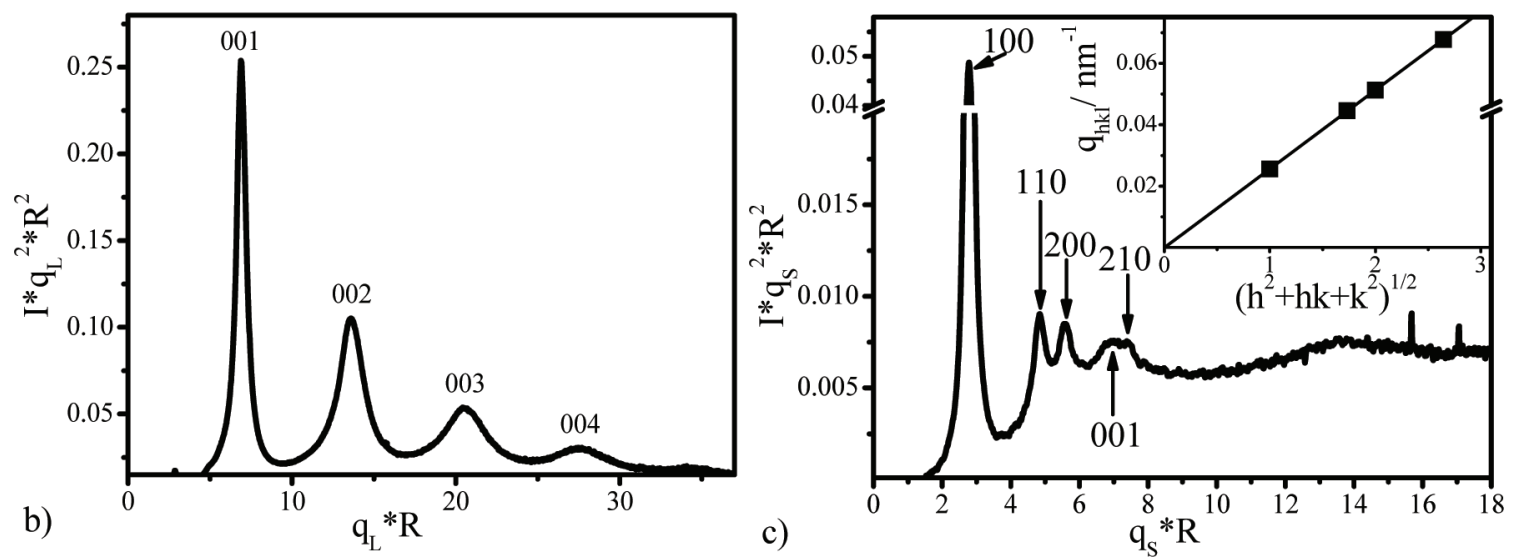

Figure 2. (a) 2D scattering pattern from an ordered gibbsite suspension of $4.8 \mathrm{vol} \%$ and the average intensity profiles for (b) $q_{\mathrm{L}}$ and (c) $q_{\mathrm{S}}$ range. The inset in panel $\mathrm{c}$ depicts the $q$ values of the Bragg reflections vs $\left(h^{2}+h k+k^{2}\right)^{1 / 2}$. The straight line indicates a very good fit of the scattering reflections to the hexagonal structure.

Smectic ordering in a hard gibbsite platelet suspension has been reported earlier. ${ }^{25}$ This occurred at a platelet concentration above columnar stability and showed a scattering pattern with a very broad hexagonal peak and two peaks in a large $q$ range. However, this scattering pattern, with disappearing hexagonal order, indicated a transition region between a columnar and a $\mathrm{Sm}_{\mathrm{A}}$ phase rather than a pure $\mathrm{Sm}_{\mathrm{B}}$ phase. Reports on a hexagonal columnar phase formed by hard gibbsite platelets sometimes show liquid order peaks at high $q$ range, ${ }^{25,41}$ that could indicate lamellar/smectic ordering. However, simple geometrical calculations using the distances from the peak positions show that an assumption of particles packing in a smectic phase would lead to a wrong volume fraction of the sample. Thus, we agree with the authors of the aforementioned publications that the peaks originate from liquid-like order within the columns and correspond to the platelet thickness. Therefore, we claim gibbsite/DMSO suspensions is the first disc-like system showing $\mathrm{Sm}_{B}$ phase formation.

Phase Diagram of Charged Platelets in Low Salinity

Regime. On the basis of the observations of the samples birefringence, we established a phase diagram of the gibbsite platelets in low salinity regime (shown in Figure 3). The suspensions are isotropic (I) up to 2.4 vol\% of platelets. Upon increasing the amount of gibbsite, we observed an $\mathrm{I} / \mathrm{Sm}_{\mathrm{B}}$ phase coexistence (from 2.4 to 3.8 vol\%) and further a $\mathrm{Sm}_{\mathrm{B}}$ phase

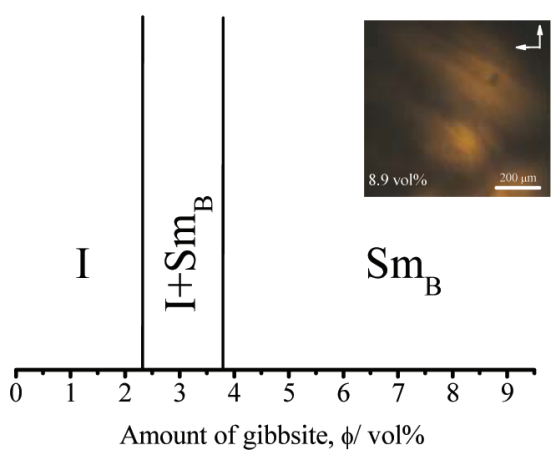

Figure 3. Phase diagram of charged colloidal platelets in low salinity regime (gibbsite in DMSO). Inset: polarization microscopy image of the $\mathrm{Sm}_{\mathrm{B}}$ optical texture. Arrows indicate polarizers' orientation. Scale bar is $200 \mu \mathrm{m}$.

(from 3.8 vol\% of gibbsite). The inset of Figure 3 contains a polarization microscopy image of the $\mathrm{Sm}_{\mathrm{B}}$ optical texture (sample with 8.9 vol\% gibbsite). Parallel lines originate from a layered lamellar order of the system and are clearly seen in the middle of the picture.

The I/LC phase transition in DMSO happens at a very low gibbsite vol\%, compared to aqueous suspensions with a small amount of added salt, where gibbsite platelets with the same D/ $L$ ratio showed an I/LC phase transition at a significantly 


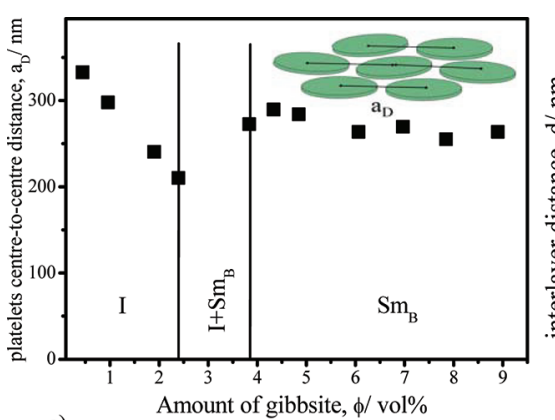

a)

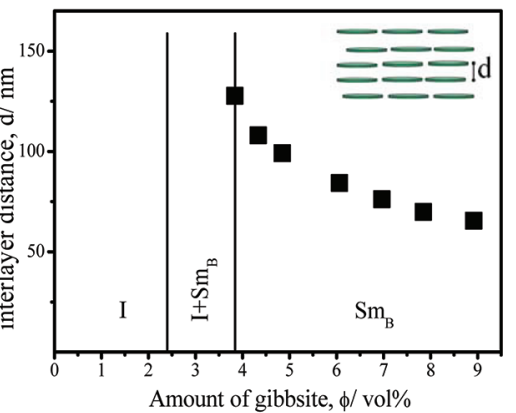

Figure 4. (a) The center-to-center distance between the platelets, $a_{\mathrm{D}}$, and (b) the interlayer distance between smectic layers, $d$, for different regions in the phase diagram.

higher amount of platelets $\left(12\right.$ vol\%). ${ }^{42}$ The low transition concentration in DMSO system is due to its low ionic strength. In aqueous systems, in addition to the small amount of salt present, both $\mathrm{CO}_{2}$ absorption and water auto proteolysis decrease the Debye screening length, thus, reducing the effective volume fraction of platelets in comparison to the DMSO dispersions.

Unfortunately, it was not possible to prepare suspensions of gibbsite platelets in DMSO with higher concentration than 9 vol\% and, thus, observe at which volume fraction the $\mathrm{Sm}_{\mathrm{B}}$ phase would end. The suspension with the high volume fraction was already very viscous and difficult to handle experimentally. Therefore, we expect that further increase of the platelets amount in this highly charged, low salinity system would lead to kinetic arrest. ${ }^{43,44}$ The structure of the arrested suspensions may lose the clear hexagonal order inside the layers. Similar observations were done on the columnar phase of hard platelets. $^{25}$

What is the reason of the $\mathrm{Sm}_{\mathrm{B}}$ phase formation in our gibbsite/DMSO suspensions? Previous studies on charged colloidal platelets with very high PDI in diameter (32\%) and a somewhat poorly pronounced shape showed an $\mathrm{I}-\mathrm{N}-\mathrm{Sm}$ (without a positional intralayer order) phase sequence in these aqueous suspensions. ${ }^{31}$ The authors explained the formation of the smectic phase by suppression of the columnar phase due to a high degree in diameter polydispersity. However, very recent work on charged graphene oxide sheets shows that a smectic phase can also be formed by platelets with much lower polydispesity in diameter (13\%). ${ }^{33}$ The diameter PDI in our system is $15.8 \%$ (Table 1 ). We have previously shown that charged gibbsite platelets with similar polydispersity are still able to show the usual for platelets $\mathrm{I}-\mathrm{N}-\mathrm{C}$ phase sequence, when dispersed in water and not in $\mathrm{DMSO},{ }^{41}$ Additionally, experimental results on hard gibbsite platelets show that for the formation of the columnar phase diameter PDI as high as $25 \%$ can be tolerated, ${ }^{25}$ which is much higher than in our system. This is why we believe that the long-range electrostatic repulsion rather than the platelets low polydispersity is a dominant driving force in the $\mathrm{SmB}$ phase formation. However, since platelet diameter polydispersity in our system is present, we believe that it prevents the system from forming a solid crystalline phase at higher volume fractions, which was predicted for platelets theoretically, ${ }^{26}$ but never found experimentally. Actually, a solid crystalline phase in rod-like colloids was observed experimentally only very recently. ${ }^{45}$

Characterization of the $\mathrm{Sm}_{\mathrm{B}}$ Phase. To further characterize the structure of the $\mathrm{Sm}_{\mathrm{B}}$ phase, we investigated the concentration dependence of the spacing parameter, $a_{\mathrm{D}}$. The center-to-center distance between the platelets, $a_{\mathrm{D}}$, in the smectic layers is always significantly smaller that the interlayer distance, $d$ (Figure 4). For all gibbsite concentrations, which showed this phase, $a_{\mathrm{D}}$ was found to fluctuate with a maximum of $70 \mathrm{~nm}$ larger than the platelets diameter $(218.4 \mathrm{~nm})$ (Figure $4 \mathrm{a})$. The $a_{\mathrm{D}}$ distance also changes only weakly with gibbsite concentration and packing of the platelets in layers only becomes about $10 \%$ higher when doubling gibbsite amount in the suspensions. On the other hand, the structure peak, which gives the most probable center-to-center distance in the isotropic phase, follows a $\phi_{\text {gibbsite }}{ }^{1 / 3}$ relation (not shown here). This is expected for isotropic systems where the mean particle distance is only affected by particle concentration. ${ }^{46,47}$ Further, the mean particle distances in the disordered phase close to the $\mathrm{I} / \mathrm{Sm}_{\mathrm{B}}$ phase transition are smaller than the $a_{\mathrm{D}}$ spacing in a smectic layer.

Since the intralayer structure in the $\mathrm{Sm}_{\mathrm{B}}$ phase changes very little with the gibbsite vol\%, the interlayer distance, $d$, should be almost directly proportional to the platelets amount. In Figure $4 \mathrm{~b}, d$ is shown for all smectic samples, and indeed, the layers are located significantly closer with increasing gibbsite concentration. The value of $d$ can be related to the Debye screening length, $\kappa^{-1}$, over the entire concentration range of the $\mathrm{Sm}_{\mathrm{B}}$ phase, with exception of the highest platelet concentration. This link is done, with the assumption that one gibbsite platelet has an effective charge of 300 . This charge density is similar to the one found for smaller gibbsite platelets in DMSO. ${ }^{48}$

The $a_{\mathrm{D}}$ spacing is significantly smaller than the sum of $D$ and the previously estimated $\kappa^{-1}$. A possible reason for this is either an enhancement of the counterions' concentration between the platelets in the layers, leading to the increased ionic strength there, that is, reduced $\kappa^{-1}$, and/or the weaker total electrostatic repulsion due to the smaller interacting surface areas. The increase of the counterion concentration in the layers would not be surprising since the intralayer volume fraction of platelets is higher than in the interlayer region. A reduced $\kappa^{-1}$ in the layers results in shorter range electrostatic interaction there than between the layers, making it possible for the platelets to pack closer edge-to-edge than face-to-face. This, we think, together with a pronounced hexagonal shape and a relatively low diameter polydispersity of the platelets, is the driving force for the hexagonal packing inside the smectic layers.

Additionally, we characterize the $\mathrm{Sm}_{\mathrm{B}}$ phase by the orientational order parameter, $P_{2}$, which was extracted from the azimuthal intensity distribution of the 001 peak (not shown here). The order parameter is low in the isotropic phase $\left(P_{2}<\right.$ 0.2 ) and lies above 0.6 in the $\mathrm{Sm}_{\mathrm{B}}$ phase for all samples. This indicates a strong ordering through the entire $\mathrm{Sm}_{\mathrm{B}}$ phase. 

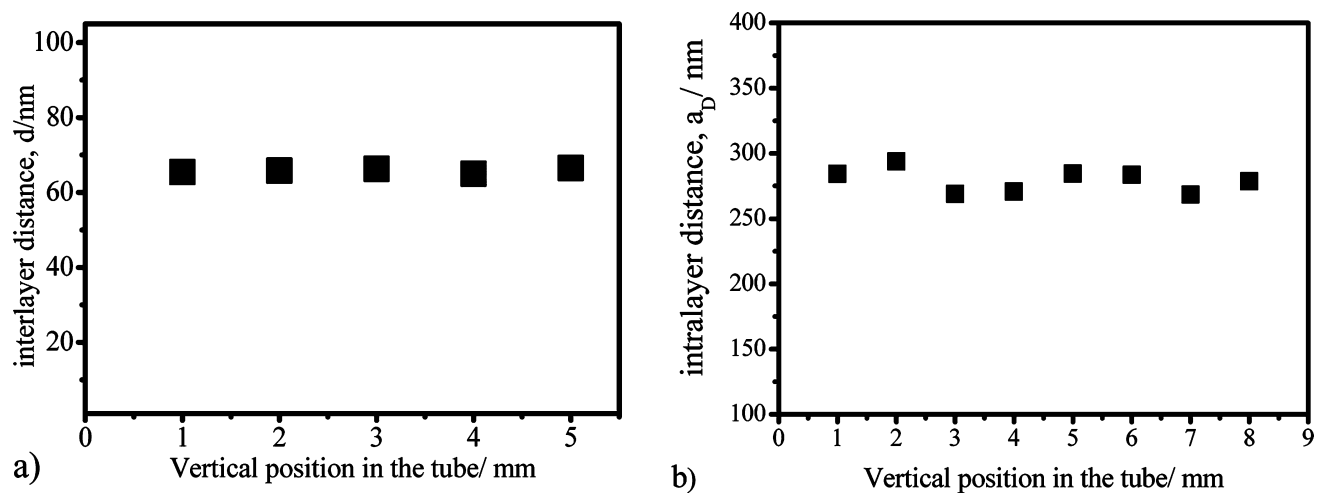

Figure 5. (a)The interlayer distance, $d$, and (b) the intralayer distance, $a_{\mathrm{D}}$, as a function of the vertical distance in the capillary, of the $S \mathrm{~m}_{\mathrm{B}} \mathrm{phase}$ in the sample with (a) 8.9 vol\% and (b) 4.8 vol\% of gibbsite.

Finally, we would like to comment on the equilibrium nature of the $\mathrm{Sm}_{\mathrm{B}}$ phase formation in gibbsite/DMSO systems. Figure 5a demonstrates that the interlayer distance, $d$, does not depend on the vertical position in the sample. Additionally, the $a_{\mathrm{D}}$ spacing does not change with the vertical distance in the capillary (Figure 5b). This means that the phase transition in gibbsite/DMSO systems is indeed in equilibrium and not affected by sedimentation. Moreover, Figure $5 \mathrm{~b}$ shows the absence of the microsegregation of large and small platelets in different layers of the $\mathrm{Sm}_{\mathrm{B}}$ phase. Such microsegregation may happen at higher platelets diameter PDI than present in this study or at higher volume fractions, when platelets are located closer to each other.

\section{CONCLUDING REMARKS}

Our experimental results unambiguously demonstrate the appearance of $\mathrm{S}_{\mathrm{B}}$ phase in the colloidal platelet system. The success of finding an unexpected $\mathrm{Sm}_{\mathrm{B}}$ phase relied upon the design of our experimental system, that of charge stabilized hexagonal platelets with a large $D / L$ ratio dispersed in a solvent with a very low ionic strength. While one can put forward intuitive arguments that by increasing the repulsion range a platelet system will have a tendency to organize in layers, that is, build a smectic phase, the fact that the intralayer hexagonal order occurs is unexpected. We speculate that the long-range repulsion and the difference in the repulsion range between platelet's faces and edges are needed to arrange a platelet system in the $\mathrm{Sm}_{\mathrm{B}}$ phase. That is why, in aqueous systems, where the salinity usually dominates over the counterion concentration, the latter does not affect $\kappa^{-1}$, making it not possible to induce the difference in the repulsive potential range. As a result, aqueous gibbsite suspensions only show the formation of $\mathrm{N}$ and $\mathrm{C}$ phases. Unfortunately, no theory or simulation results are available that describe the formation of any smectic phase by the disc- or platelet-like species. However, our study shows that manipulating interactions leads to a new richness in the phase behavior of colloids.

\section{AUTHOR INFORMATION}

\section{Corresponding Author}

D.Kleshchanok@uu.nl

\section{Notes}

The authors declare no competing financial interest.

\section{ACKNOWLEDGMENTS}

Rik Wensink, Eric Grelet, Andrei Petukhov and Adrian Rennie are thanked for many fruitful discussions. The team of BM-26 is thanked for the technical assistance during the synchrotron experiments. This work is part of the research program of the Foundation for Fundamental Research on Matter (FOM), which is part of The Netherlands Organization for Scientific Research (NWO). The beam time was kindly provided by NWO.

\section{REFERENCES}

(1) Grzybowski, B. A.; Wilmer, C. E.; Kim, J.; Browne, K. P.; Bishop, K. J. M. Soft Matter 2009, 5, 1110-1128.

(2) Li, F.; Josephson, D. P.; Stein, A. Angew. Chem., Int. Ed. 2011, 50, 360-388.

(3) Pusey, P. N.; van Megen, W. Nature 1986, 320, 340-342.

(4) Poon, W. C. K.; Pusey, P. N. In Observation, Prediction and Simulation of Phase Transitions in Complex Fluids; Baus, M., Rull, L. F., Ryckaert, J. P., Eds.; Kluwer Academic: Dordrecht, 1995; pp 3-51.

(5) Frenkel, D. In Liquids, Freezing and Glass Transition; Hansen, J. P., Levesque, D., Zinn-Justin, J., Eds.; Elsevier Science: Amsterdam, 1991; pp 689-762.

(6) Glotzer, S. C.; Solomon, M. J. Nat. Mater. 2007, 6, 557-562.

(7) Davidson, P.; Gabriel, J.-C. P. Curr. Opin. Colloid Interface Sci. 2005, 9, 377-383.

(8) Min, Y. J.; Akbulut, M.; Kristiansen, K.; Golan, Y.; Israelachvili, J. Nat. Mater. 2008, 7, 527-538.

(9) Adams, M.; Dogic, Z.; Keller, S. L.; Fraden, S. Nature 1998, 393, 349-352.

(10) Rossi, L.; Sacanna, S.; Irvine, W. T. M.; Chaikin, P. M.; Pine, D. J.; Philipse, A. P. Soft Matter 2011, 7, 4139-4142.

(11) Barry, E.; Dogic, Z. Proc. Natl. Acad. Sci. U.S.A. 2010, 107, 10348-10353.

(12) Mason, T. G. Phys. Rev. E 2002, 66, 060402.

(13) Shevchenko, E. V.; Talapin, D. V.; Kotov, N. A.; O’Brien, S.; Murray, C. B. Nature 2006, 439, 55-59.

(14) Miszta, K.; de Graaf, J.; Bertoni, G.; Dorfs, D.; Brescia, R.; Marras, S.; Ceseracciu, L.; Cingolani, R.; van Roij, R.; Dijkstra, M.; Manna, L. Nat. Mater. 2011, 10, 872-876.

(15) Leunissen, M. E.; Christova, C. G.; Hynninen, A. P.; Royall, C. P.; Campbell, A. I.; Imhof, A.; Dijkstra, M.; van Roij, R.; van Blaaderen, A. Nature 2005, 437, 235-240.

(16) Lahiri, J.; Isaacs, L.; Grzybowski, B.; Carbeck, J. D.; Whitesides, G. M. Langmuir 1999, 15, 7186-7198.

(17) Kalsin, A. M.; Fialkowski, M.; Paszewski, M.; Smoukov, S. K.; Bishop, K. J. M.; Grzybowski, B. A. Science 2006, 312, 420-424.

(18) Sacanna, S.; Irvine, W. T. M.; Chaikin, P. M.; Pine, D. J. Nature 2010, 464, 575-578. 
(19) Alivisatos, A. P.; Johnsson, K. P.; Peng, X. G.; Wilson, T. E.; Loweth, C. J.; Bruchez, M. P.; Schultz, P. G. Nature 1996, 382, 609611.

(20) Jones, M. R.; Macfarlane, R. J.; Lee, B.; Zhang, J.; Young, K. L.; Senesi, A. J.; Mirkin, C. A. Nat. Mater. 2010, 9, 913-917.

(21) Mitchell, G. P.; Mirkin, C. A.; Letsinger, R. L. J. Am. Chem. Soc. 1999, 121, 8122-8123.

(22) Macfarlane, R. J.; Lee, B.; Jones, M. R.; Harris, N.; Schatz, G. C.; Mirkin, C. A. Science 2011, 334, 204-208.

(23) Peroukidis, S. D.; Vanakaras, A. G.; Photinos, D. J. J. Mater. Chem. 2010, 20, 10495.

(24) Wensink, H. H.; Lekkerkerker, H. N. W. Mol. Phys. 2009, 107, 2111-2118.

(25) van der Kooij, F. M.; Kassapidou, K.; Lekkerkerker, H. N. W. Nature 2000, 406, 868-871.

(26) Veerman, J. A. C.; Frenkel, D. Phys. Rev. A 1992, 45, 56325648.

(27) Onsager, L. Ann. N.Y. Acad. Sci. 1949, 51, 627.

(28) Mourad, M. C. D.; Byelov, D. V.; Petukhov, A. V.; de Winter, D. A. M.; Verkleij, A. J.; Lekkerkerker, H. N. W. J. Phys. Chem. B 2009, 113, 11604-11613.

(29) Gabriel, J. C. P.; Camerel, F.; Lemaire, B. J.; Desvaux, H.; Davidson, P.; Batail, P. Nature 2001, 413, 504-508.

(30) Wang, N.; Liu, S. Y.; Zhang, J.; Wu, Z. H.; Chen, J.; Sun, D. J. Soft Matter 2005, 1, 428-430.

(31) Sun, D. Z.; Sue, H. J.; Cheng, Z. D.; Martinez-Raton, Y.; Velasco, E. Phys. Rev. E 2009, 80.

(32) Xu, Z.; Gao, C. ACS Nano 2011, 5, 2908-2915.

(33) Xu, Z.; Gao, C. Nat. Commun. 2011, 2, 571.

(34) Morales-Anda, L.; Wensink, H. H.; Galindo, A.; Gil-Villegas, A. J. Chem. Phys. 2012, 136, 034901.

(35) Oswald, P.; Pieranski, P. Smectc and Columnar Liquid Crystals; Taylor\&Francis Group: Boca Raton, FL, 2006.

(36) Kuijk, A. Fluorescent colloidal silica rods-synthesis and phase behavior, Ph.D. Thesis, Utrecht University, 2012.

(37) Wierenga, A. M.; Lenstra, T. A. J.; Philipse, A. P. Colloids Surf., A 1998, 134, 359-371.

(38) Borsboom, M.; Bras, W.; Cerjak, I.; Detollenaere, D.; Glastra van Loon, D.; Goedtkindt, P.; Konijnenburg, M.; Lassing, P.; Levine, Y. K.; Munneke, B.; Oversluizen, M.; van Tol, R.; Vlieg, E. J. Synchrotron Radiat. 1998, 5, 518-520.

(39) Petukhov, A. V.; Thijssen, J. H. J.; t Hart, D. C.; Imhof, A.; van Blaaderen, A.; Dolbnya, I. P.; Snigirev, A.; Moussaid, A.; Snigireva, I. J. Appl. Crystallogr. 2006, 39, 137-144.

(40) Hahn, T. International Tables for Crystallography; Reidel: Dortrecht, 1983.

(41) van der Beek, D.; Petukhov, A. V.; Oversteegen, S. M.; Vroege, G. J.; Lekkerkerker, H. N. W. Eur. Phys. J. E 2005, 16, 253-258.

(42) Kleshchanok, D.; Meijer, J. M.; Petukhov, A. V.; Portale, G.; Lekkerkerker, H. N. W. Soft Matter 2012, 8, 191-197.

(43) Michot, L. J.; Bihannic, I.; Maddi, S.; Funari, S. S.; Baravian, C.; Levitz, P.; Davidson, P. Proc. Natl. Acad. Sci. U.S.A. 2006, 103, 1610116104.

(44) Tanaka, H.; Meunier, J.; Bonn, D. Phys. Rev. E. 2004, 69, 031404.

(45) Grelet, E. Phys. Rev. Lett. 2008, 100, 168301.

(46) Heinen, M.; Holmqvist, P.; Banchio, A. J.; Nägele, G. J. Chem. Phys. 2011, 134, 129901.

(47) Heinen, M.; Holmqvist, P.; Banchio, A. J.; Nägele, G. J. Chem. Phys. 2011, 134, 044532.

(48) Kleshchanok, D.; Heinen, M.; Nägele, G.; Holmqvist, P. Soft Matter 2012, 8, 1584-1592. 